\title{
Progesterone at encoding predicts subsequent emotional memory
}

\author{
Nicole Ertman, ${ }^{1,3}$ Joseph M. Andreano, ${ }^{1,2}$ and Larry Cahill ${ }^{1}$ \\ ${ }^{1}$ Center for the Neurobiology of Learning and Memory, Department of Neurobiology and Behavior, University of California, Irvine, \\ California 92697, USA; ${ }^{2}$ Department of Psychiatry, Massachusetts General Hospital, Charlestown, Massachusetts 02139, USA
}

\begin{abstract}
Significant sex differences in the well-documented relationship between stress hormones and memory have emerged in recent studies. The potentiating effects of glucocorticoids on memory vary across the menstrual cycle, suggesting a potential interaction between these stress hormones and endogenously cycling sex hormones. Here, we show that memory for emotional materials changes significantly in accordance with hormonal changes across the menstrual cycle, suggesting that ovarian sex hormones influence the modulation of emotional memories. Sixty healthy, naturally cycling women rated 120 images on arousal and valence. One week later they completed free recall and recognition memory tests. Their menstrual cycle phases were estimated by self-report and confirmed by salivary assay of $17 \beta$-estradiol and progesterone. Memory for emotional items only was significantly better in the high hormone (luteal) phase compared with the low hormone (follicular) phase on the free recall test; on both tests memory correlated positively with progesterone collected at the time of encoding. These findings suggest that emotional memory performance changes across the menstrual cycle, and that this change is in part mediated by endogenous progesterone cycling.
\end{abstract}

A significant body of literature has demonstrated that sex hormones influence cognition (Kimura and Hampson 1994; Sherwin 1994; Van Goozen et al. 1995; Kimura 1996; Slabbekorn et al. 1999; van Wingen et al. 2008a) and, further, it has been suggested that the endogenous sex hormone fluctuations associated with the menstrual cycle can also have cognitive effects (Hampson 1990; Andreano et al. 2008). The menstrual cycle is characterized by changes in ovarian sex hormones, including the neuroactive steroids $17 \beta$-estradiol and progesterone. The onset of menses begins in the follicular phase, during which these hormones are relatively lowered, until late in the second week of the cycle, shortly before ovulation. Estrogens swiftly increase and then plummet after ovulation. Following ovulation, during the luteal phase, estrogens remain high relative to the early follicular phase, and progesterone increases significantly.

During the luteal phase of the menstrual cycle, when estrogens and progesterone are elevated relative to the early follicular phase, memory in multiple mnemonic domains is enhanced. Superior performance in the luteal phase has been shown on tasks of conceptual implicit memory (Maki et al. 2002), visual memory (Phillips and Sherwin 1992), and verbal memory (Rosenberg and Park 2002). Conversely, when ovarian sex hormones are decreased during the early follicular phase, better spatial memory has been observed in both humans (Postma et al. 1999; Hausmann et al. 2000) and rhesus monkeys (Lacreuse et al. 2001).

Limited evidence (reviewed below) has implied that emotional memory may also be subject to influences by sex hormones, although to our knowledge this hypothesis had not been explicitly tested prior to this investigation. Emotional memory has been shown by a tremendous body of research to depend on stress hormones (for reviews, see McGaugh 2000; Roozendaal 2000; LaBar and Cabeza 2006) such that increased activity of sympathetic (Cahill and Alkire 2003; van Stegeren 2008) and adrenocortical (Buchanan and Lovallo 2001; Abercrombie et al. 2003; Cahill et al. 2003) hormones in the amygdala enhance memory.

\footnotetext{
${ }^{3}$ Corresponding author.
}

E-mail nertman@uci.edu.

Article is online at http://www.learnmem.org/cgi/doi/10.1101/lm.023267.111.
In humans, cortisol has been shown to dose dependently influence memory encoding, such that small to moderate increases in cortisol improve memory, and beyond a critical point further increases in cortisol decreased memory (Domes et al. 2005; Andreano and Cahill 2006). An enhancing effect of cortisol on memory has been demonstrated using both endogenous (Andreano and Cahill 2006) and exogenous (Buchanan and Lovallo 2001) glucocorticoid administration. Additionally, norepinephrine is both strongly associated with emotional memory (McGaugh and Roozendaal 2002; van Stegeren et al. 2008; Segal and Cahill 2009) and is significantly higher at baseline in the luteal relative to the follicular phase (Minson et al. 2000).

Important sex differences have been observed in the mnemonic influences of these stress hormones. Cortisol administration impaired associative learning and reduced regional cerebral blood flow in the anterior cingulate, lateral orbitofrontal cortex, and medial prefrontal cortex in men, while enhancing performance and increasing blood flow in the same regions in women (Stark et al. 2006). In another study, post-training cortisol levels correlated positively with learning only in men, with no discernable relationship between cortisol and learning in women (Zorawski et al. 2006). However, menstrual cycle position was not considered in either study. While Kuhlmann and Wolf (2005) found no differences in the effects of cortisol on retrieval across the menstrual cycle, Andreano et al. (2008) showed in a separate investigation that the relationship between memory retrieval and cortisol at the time of encoding does appear to be dependent on menstrual-cycle position. Women in the early follicular phase of the menstrual cycle show no relationship whatsoever between cortisol and memory, and women in the mid-luteal phase show a highly significant positive correlation between cortisol and memory. This finding, in particular, suggests that in women stress hormones and sex hormones interact to produce the emotional memory enhancement that has been robustly and repeatedly observed throughout the literature.

Because of evidence that emotional material is better remembered due to stress hormone release, and that the relationship between stress hormones and memory changes across the menstrual cycle, we hypothesized that emotional memory 
Table 1. Salivary sex hormone concentrations

\begin{tabular}{lcc}
\hline & $\begin{array}{c}\text { Salivary } 17 \beta \text {-estradiol } \\
\text { mean } \pm \text { SD }(\mathbf{p g} / \mathbf{m L})\end{array}$ & $\begin{array}{c}\text { Salivary progesterone } \\
\text { mean } \pm \text { SD }(\mathbf{p g} / \mathbf{m L})\end{array}$ \\
\hline Follicular group & $2.76 \pm 1.11$ & $47.9 \pm 42.0$ \\
Luteal group & $3.68 \pm 1.86^{\mathrm{a}}$ & $82.9 \pm 50.9^{\mathrm{b}}$ \\
\hline
\end{tabular}

Salivary $17 \beta$-estradiol and progesterone concentrations are both significantly higher in the luteal group relative to the follicular group.

${ }^{a} P<0.05$.

${ }^{\mathrm{b}} \mathrm{p}<0.01$.

performance may change across the menstrual cycle. This hypothesis is supported by findings from animal literature: Rats in the high-hormone proestrus phase of the estrous cycle performed worse on a spatial-contextual conditioning task relative to both male rats and female rats in the estrus phase (Markus and Zecevic 1997). Similarly, Warren and Juraska (1997) found impaired performance on a spatial task in proestrus relative to estrus. However, in the same study, proestrus females performed better than those in estrus on a cue memory task, and in a separate investigation, proestrus females acquired a conditioned fear response more quickly than those in other stages (Shors et al. 1998). These findings suggest that any mnemonic advantages seen across the menstrual cycle may be selective for particular memory systems.

Here, we investigate the influences of the menstrual cycle on the emotional memory system in humans. In this study we provide the first evidence of which we are aware that in a classic emotional memory task, retention of emotional stimuli changes significantly across the menstrual cycle, and these changes correlate with progesterone levels.

Seventy-two naturally cycling women were recruited from the University of California, Irvine undergraduate population. Participants ranged in age from 18 to $28(M=19.79 \mathrm{yr}, S D=$ 1.97). Written informed consent was obtained from all subjects in accordance with the University of California, Irvine's Institutional Review Board. Twelve participants were excluded from analysis: five reported cycles without menses in the past $2 \mathrm{mo}$, one reported recent use of hormonal contraception, four failed to produce a viable saliva sample, and two failed to complete the task as instructed.

To perform a group-level analysis, participants were sorted post hoc into two hormonally distinct groups that corresponded approximately to the follicular and luteal phases of the menstrual cycle based on a brief interview administered by the experimenter. Participants undergoing the encoding task on days $0-14$ were placed in the low-hormone follicular group $(n=36)$ and those doing so on days 15-31 $(n=24)$ comprised the high hormone luteal group. Hormone analysis using Salimetrics (State College) enzyme immunoassays of $17 \beta$-estradiol and progesterone confirmed that the two groups differed significantly in both hormones. A one-way ANOVA demonstrated that both $17 \beta$-estradiol and progesterone were significantly higher in the luteal phase relative to the follicular phase (respectively: $F_{(1,58)}=5.64, P=$ 0.02 and $F_{(1,58)}=8.39, P=0.0053$ ) (Table 1 ). Although we anticipated a third, high-estrogen, low-progesterone group corresponding approximately to the late follicular phase, zero participants were found to have this hormonal profile.

The memory task was comprised of an encoding phase followed by retrieval tests $7 \mathrm{~d}$ later, a retention interval consistent with previous investigations from our laboratory (Andreano and Cahill 2006; Andreano et al. 2008) and others (Buchanan and Lovallo 2001). At the onset of each encoding session the experimenter obtained informed consent from the participant. The participant then completed the Positive Affect Negative Affect
Schedule (PANAS), followed by the collection of a 2-mL saliva sample using the "passive drool" method in which participants expectorate directly into a sterile Falcon tube. This sample was immediately frozen at $-20^{\circ} \mathrm{C}$ and later used to perform the previously reported $17 \beta$-estradiol and progesterone assays.

The encoding task itself consisted of 120 images selected from the International Affective Picture System (IAPS) (Lang et al. 2008). Although subjective ratings were used for all analyses, the images were selected so that according to IAPS normative ratings, 60 were both negatively valenced $(M=2.66, S D=0.88)$ and arousing $(M=5.73, S D=0.55)$, and 60 were neutrally valenced $(M=4.78, S D=0.80)$ and non-arousing $(M=3.43, S D=0.63)$. Slides were viewed for $3 \mathrm{sec}$ each. After a 1-sec delay, participants rated the arousal $(M=4.77, S D=1.27$, all slides, all participants) and then valence $(M=3.82, S D=0.57$, all slides, all participants) of each image using a 9-point Likert scale. One week later, participants returned to the laboratory for memory testing. They first completed an untimed free recall test during which they were instructed to write down a brief description of as many slides as they could remember and then to provide any additional details they could recall about each slide. Slides were scored as correctly recalled if the rater could identify the slide based on the participant's description and unambiguously distinguish it from all other images presented.

A one-way ANOVA revealed significantly higher total recall in the luteal phase relative to the follicular phase $\left(F_{(1,58)}=8.96\right.$, $P=0.004$ ) (Fig. 1). A two-way mixed model ANOVA revealed a significant main effect of phase, $F_{(1,57)}=8.37, P=0.004$, and a highly significant main effect of emotionality, $F_{(1,57)}=32.67$, $P<0.0001$ without a significant interaction term (Fig. 2). As shown in Figure 2, the superior memory performance by the luteal group appears to have been driven primarily by better memory for emotional slides, $F_{(1,58)}=5.73, P=0.02$, as memory for neutral slides did not differ between the two groups, $F_{(1,58)}=2.82, P=$ 0.10 . The effect size of the difference in emotional recall for the two groups was moderate, $d=0.52$.

Salivary progesterone concentrations significantly correlated with emotional, $r_{(58)}=0.29, P=0.03$ (Fig. 3), but not neutral, $r_{(58)}=-0.12, P=0.36$ free recall scores. These two correlations were found to be significantly different by Fisher's $\mathrm{Z}$ test $(P=$ $0.03)$. Salivary $17 \beta$-estradiol had no relationship with either emotional, $r_{(58)}=0.04, P=0.73$ or neutral, $r_{(58)}=0.13, P=0.31$ recall.

Following the recall test, participants were asked to complete a recognition test in which the 120 encoding slides were randomly interspersed with an equivalent number of foil slides also selected from IAPS matched for normative arousal and valence. After each slide presentation, participants indicated whether or not they remembered seeing it before and then rated their confidence in this memory on a scale of 1 to 9 .

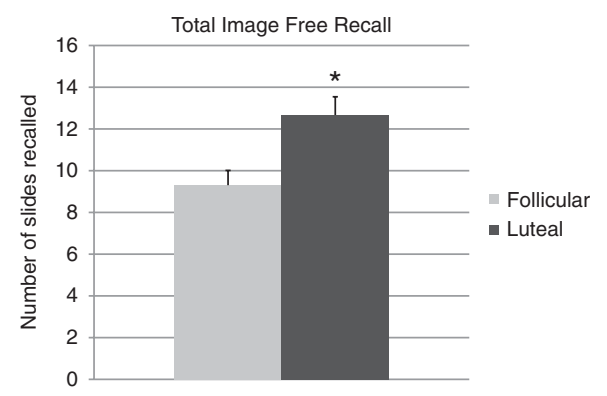

Figure 1. Total image free recall. Total recall is significantly better in the luteal phase relative to the follicular phase. $(*) P<0.01$. 


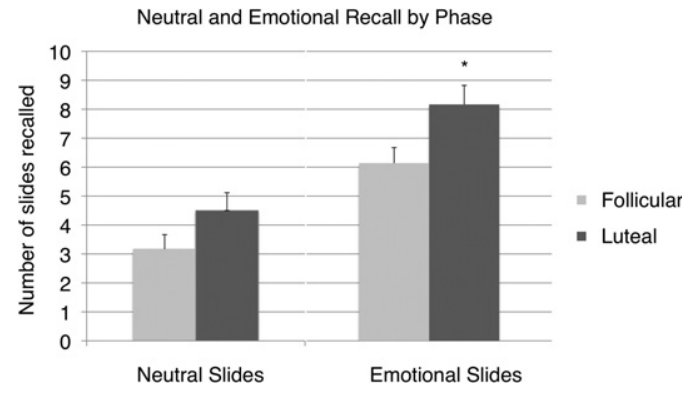

Figure 2. Neutral and emotional recall by phase. The luteal free recall memory enhancement is only significant for emotional slides. (*) $P<0.05$.

Consistent with performance on the recall task, correlations between sex hormones and recognition scores showed a significant positive relationship between emotional hits and progesterone, $r_{(58)}=0.31, P=0.02$ (Fig. 3) but not $17 \beta$-estradiol, $r_{(58)}=$ $0.19, P=0.16$. Group differences in performance on this task did not reach statistical significance, potentially due to a ceiling effect: participants correctly identified, on average, $87 \%$ of slides with accuracy ([hits + correct rejections]/total slides) ranging from $70 \%$ to $96 \%$.

The present results suggest that retention of emotional material changes significantly over the course of the menstrual cycle as ovarian sex hormones fluctuate. Memory for stimuli subjectively rated as emotionally arousing was significantly better than that for neutral stimuli across the entire menstrual cycle; however, during the high-hormone phase this memory enhancement increased still further. Regardless of menstrual cycle phase, emotional memory correlated positively with circulating levels of progesterone as measured by salivary progesterone concentrations. A positive relationship between progesterone, but not $17 \beta$-estradiol levels and emotional memory was found in both free recall and recognition memory tests. Although $17 \beta$-estradiol levels did not correlate with any memory scores, the possibility that progesterone's effects depend in part on estrogenic activity cannot be eliminated.

These findings fit well with previous data suggesting a potentiating effect of progesterone on emotional memory, evidenced by a positive relationship between progesterone and intrusive recollections after an emotional event (Ferree et al. 2011) on cortisol's relationship to emotional memory (Andreano et al. 2008) and on amygdala responsiveness to emotional stimuli (van Wingen et al. 2008b; Andreano and Cahill 2010). Exogenous administration of luteal levels of progesterone has been associated with significant increases in activity in the right amygdala and, to a lesser extent, the left amygdala (van Wingen et al. 2008b). Because subsequent memory for emotional stimuli is associated with functional activation of the amygdala (Canli et al. 2000; Cahill et al. 2004), our behavioral findings of a relationship between progesterone and emotional memory accord well with the existing imaging data.

However, existing behavioral investigations of progesterone's effects on other memory systems did not predict enhancing effects on emotional memory. In an investigation of verbal memory, high doses of exogenously administered progesterone impaired immediate verbal recall (Freeman et al. 1992) and facial recognition (van Wingen et al. 2008a). Similarly, the progesterone metabolite allopregnanolone has been shown to impair episodic memory (Kask et al. 2008). Importantly, however, these findings are based on progesterone levels $\sim 10$-fold higher than those observed in the luteal phase, suggesting that progesterone's cognitive effects may be dose dependent. Further, our findings appear to be selective for emotional material, suggesting that progesterone's effects may also differ by memory system.

Several predictions for future research could be extrapolated from these data. The sensitivity of the hypothalamic-pituitaryadrenal (HPA) axis changes significantly across the menstrual cycle, such that women in the luteal phase have a significantly higher cortisol response to both psychosocial stressors and adrenocorticotropic hormone stimulation compared with women in the follicular phase (Kirschbaum et al. 1999). Further, the action of the stress hormone cortisol depends on levels of circulating sex hormones (Andreano et al. 2008). Because previous literature has emphasized the importance of stress hormones in consolidation of emotional memories, which were not accounted for in this study, future investigation of differences in emotional memory across the menstrual cycle would benefit from the assessment of both types of hormones.

Studies using similar tasks (van Stegeren et al. 2008) have not shown a cortisol response without administration of hydrocortisone (Buchanan and Lovallo 2001; Abercrombie et al. 2003; Kuhlmann and Wolf 2006), suggesting that the emotional memory enhancement in the present experiment is likely driven at least in part by adrenergic activation, which was shown to account for over $50 \%$ of the observed variance in free recall (Segal and Cahill 2009). Because we observed a change in memory across the menstrual cycle in the almost certain absence of a cortisol increase, and because plasma levels of adrenergic hormones are subject to changes across the menstrual cycle (Minson et al. 2000), it may be the case that progesterone potentiates the
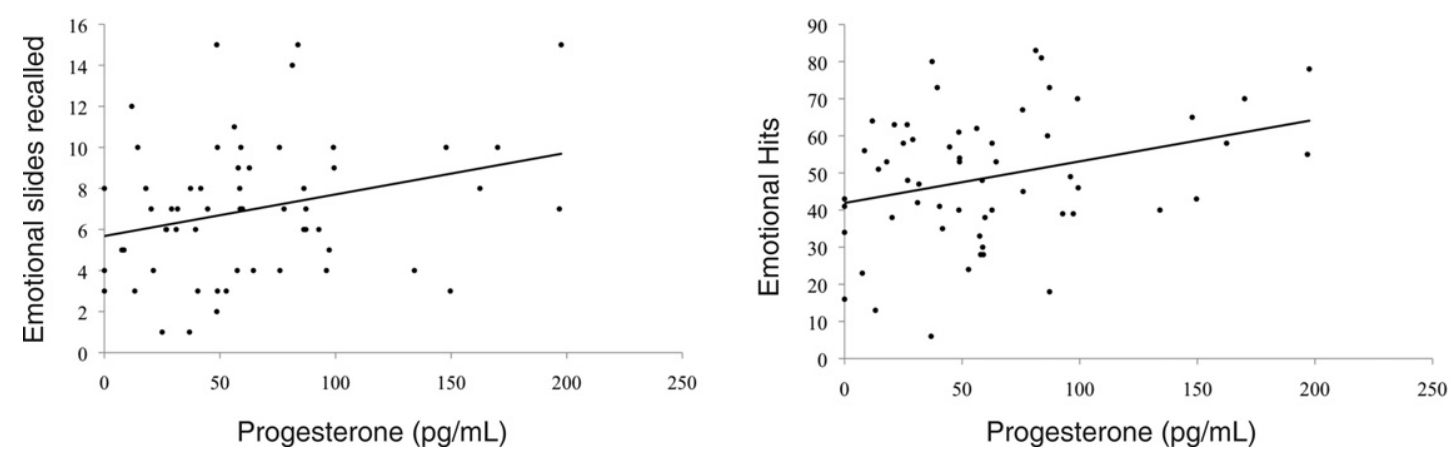

Figure 3. Emotional recall and recognition memory correlate positively with salivary progesterone at the time of encoding, $r=0.29, P=0.03$ and $r=0.31, P=0.02$, respectively. 
sympathetic nervous response and/or the action of adrenergic neurotransmitters. Therefore, a more complete model of emotional memory enhancement in women that accounts for more of the observed variance may be contingent on assessing the activity of and potential interactions between the specific sex and stress hormones implicated in this type of task-i.e., both progesterone and norepinephrine.

The present findings, considered in the context of previous literature, suggest that sex hormones and stress hormones may influence memory both separately and together. Future studies are needed to better characterize the relationship between these two categories of hormones. It may be of particular interest to differentiate between encoding and retrieval effects, as glucocorticoids have been shown to have opposing effects, depending on which stage of the memory task they were administered. In both men and women, glucocorticoid administration is associated with a dose-dependent memory enhancement at encoding (Buchanan and Lovallo 2001; Cahill et al. 2003), but memory impairment when administered shortly before retrieval (de Quervain et al. 2000; Kuhlmann and Wolf 2005; Kuhlmann et al. 2005). Here, we have demonstrated a significant relationship between memory and progesterone at the time of encoding; however, this does not preclude a possible effect (either impairing or enhancing) of sex hormones on retrieval. This is an important possibility that should be explored in future investigations.

Characterizing the relationship between sex hormones, stress hormones, and memory is of particular interest due to significant sex differences in the prevalence of mood disorders, some of which are associated with changes to brain regions critical to emotional memory (Nestler et al. 2002; Drevets 2003; Ressler and Mayberg 2007) as well as a disrupted stress response (Arborelius et al. 1999). Specifically, major depression (Burke et al. 2005) and post-traumatic stress disorder (PTSD) both involve abnormal cortisol profiles (Yehuda et al. 1990, 1995, 1996), and both disorders are more prevalent in women than in men (Kessler et al. 1994, 1995). Recent findings from our laboratory suggest a relationship between menstrual cycle and intrusive recollections for emotional stimuli (Ferree et al. 2011), which serve as a laboratory analog for intrusive memories, a hallmark of PTSD (Reynolds and Brewin 1998). Women in the luteal phase of the menstrual cycle report significantly more intrusive recollections than those in the follicular phase, and these intrusions correlate positively with progesterone at the time of encoding. In addition to these effects on mood disorders, interacting effects of declining sex hormones and altered HPA axis activity may also be important to understanding age-related cognitive decline (for review, see Conrad and Bimonte-Nelson 2010).

In this study we observed that retention of emotional memories increases when stimuli are presented during the luteal phase of the menstrual cycle. Further, both recall and recognition memory for emotional stimuli correlates positively with progesterone. We consider these findings to be of potential importance to characterizing the interactions between sex and stress hormones, which may in turn clarify the etiology of those disorders affected by both sex and stress.

\section{Acknowledgments}

This research was supported by NIMH grant R01MH057508 to L.C.

\section{References}

Abercrombie HC, Kalin NH, Thurow ME, Rosenkranz MA, Davidson RJ. 2003. Cortisol variations in humans affects memory for emotionally laden and neutral information. Behav Neurosci 117: 505-516.
Andreano JM, Cahill L. 2006. Glucocorticoid release and memory consolidation in men and women. Psychol Sci 17: 466-470.

Andreano JM, Cahill L. 2010. Menstrual cycle modulation of medial temporal activity evoked by negative emotion. NeuroImage 53: $1286-1293$.

Andreano JM, Arjomandi H, Cahill L. 2008. Menstrual cycle modulation of the relationship between cortisol and long-term memory. Psychoneuroendocrinology 33: 874-882.

Arborelius L, Owens MJ, Plotsky PM, Nemeroff CB. 1999. The role of corticotropin-releasing factor in depression and anxiety disorders. I Endocrinol 160: 1-12.

Buchanan TW, Lovallo WR. 2001. Enhanced memory for emotional material following stress-level cortisol treatment in humans. Psychoneuroendocrinology 26: 307-317.

Burke HM, Davis MC, Otte C, Mohr DC. 2005. Depression and cortisol responses to psychological stress: A meta-analysis. Psychoneuroendocrinology 30: 846-856.

Cahill L, Alkire MT. 2003. Epinephrine enhancement of human memory consolidation: Interaction with arousal at encoding. Neurobiol Learn Mem 79: 194-198.

Cahill L, Gorski L, Le K. 2003. Enhanced human memory consolidation with post-learning stress: Interaction with the degree of arousal at encording. Learn Mem 10: 270-274.

Cahill L, Uncapher M, Kilpatrick L, Alkire MT, Turner J. 2004. Sex-related hemispheric lateralization of amygdala function in emotionally influenced memory: An fMRI investigation. Learn Mem 11: 261-266.

Canli T, Zhao Z, Brewer J, Gabrieli JD, Cahill L. 2000. Event-related activation in the human amygdala associates with later memory for individual emotional experiences. J Neurosci 20: RC99 (1-5).

Conrad CD, Bimonte-Nelson HA. 2010. Impact of the hypothalamic-pituitary-adrenal/gonadal axes on trajectory of age-related cognitive decline. Prog Brain Res 182: 31-76.

de Quervain DJ, Roozendaal B, Nitsch RM, McGaugh JL, Hock C. 2000. Acute cortisone administration impairs retrieval of long-term declarative memory in humans. Nat Neurosci 3: 313-314.

Domes G, Rothfischer J, Reichwald U, Hautzinger M. 2005. Inverted-U function between salivary cortisol and retrieval of verbal memory after hydrocortisone treatment. Behav Neurosci 119: 512-517.

Drevets W. 2003. Neuroimaging abnormalities in the amygdala in mood disorders. Ann N Y Acad Sci 985: 420-444.

Ferree NK, Kamat R, Cahill L. 2011. Influences of menstrual cycle position and sex hormone levels on spontaneous intrusive recollections following emotional stimuli. Conscious Cogn doi: 10.1016/concog. 2011.02.003.

Freeman EW, Weinstock L, Rickels K, Sondheimer SJ, Coutifaris C. 1992. A placebo-controlled study of effects of oral progesterone on performance and mood. Br J Clin Pharmacol 33: 293-298.

Hampson E. 1990. Variations in sex-related cognitive abilities across the menstrual cycle. Brain Cogn 14: 26-43.

Hausmann M, Slabbekorn D, van Goozen SHM, Cohen-Kettenis PT, Güntürkün O. 2000. Sex hormones affect spatial abilities during the menstrual cycle. Behav Neurosci 114: 1245-1250.

Kask K, Bäckström T, Nilsson L, Sundström-Poromaa I. 2008. Allopregnanolone impairs episodic memory in healthy women. Psychopharmacology 199: 161-168.

Kessler RC, McGonagle KA, Zhao S, Nelson CB, Hughes M, Eshleman S, Wittchen H, Kendler KS. 1994. Lifetime and 12-month prevalence of DSM-III-R psychiatric disorders in the United States. Arch Gen Psychiatry 51: $355-364$.

Kessler RC, Sonnega A, Bromet E, Hughes M, Nelson CB. 1995. Posttraumatic stress disorder in the National Comorbidity Survey. Arch Gen Psychiatry 52: 1048-1060.

Kimura D. 1996. Sex, sexual orientation and sex hormones in human cognitive function. Curr Opin Neurobiol 6: 259-263.

Kimura D, Hampson E. 1994. Cognitive pattern in men and women is influenced by fluctuations in sex hormones. Curr Dir Psychol Sci 3: $57-61$.

Kirschbaum C, Kudielka BM, Gaab J, Schommer NC, Hellhammer DH. 1999. Impact of gender, menstrual cycle phase, and oral contraceptives on the activity of the hypothalamus-pituitary-adrenal axis. Psychosom Med 61: $154-162$.

Kuhlmann S, Wolf OT. 2005. Cortisol and memory retrieval in women: Influence of menstrual cycle and oral contraceptives. Psychopharmacology 183: 65-71.

Kuhlmann S, Wolf OT. 2006. Arousal and cortisol interact in modulating memory consolidation in healthy young men. Behav Neurosci 120: $217-223$.

Kuhlmann S, Kirschbaum C, Wolf OT. 2005. Effects of oral cortisol treatment in healthy young women on memory retrieval of negative and neutral words. Neurobiol Learn Mem 83: 158-162.

LaBar KS, Cabeza R. 2006. Cognitive neuroscience of emotional memory. Nat Rev Neurosci 7: 54-64. 
Lacreuse A, Verreault M, Herndon JG. 2001. Fluctuations in spatial recognition memory across the menstrual cycle in female rhesus monkeys. Psychoneuroendocrinology 26: 623-639.

Lang PJ, Bradley MM, Cuthbert BN. 2008. International affective picture system (IAPS): Affective ratings of pictures and instruction manual. Technical Report A-8. University of Florida, Gainesville, FL.

Maki PM, Rich JB, Rosenbaum RS. 2002. Implicit memory varies across the menstrual cycle: Estrogen effects in young women. Neuropsychologia 40: $518-529$.

Markus EJ, Zecevic M. 1997. Sex differences and estrous cycle changes in hippocampus-dependent fear conditioning. Psychobiology 25: 246-252.

McGaugh JL. 2000. Memory-a century of consolidation. Science 287: 248-251.

McGaugh JL, Roozendaal B. 2002. Role of adrenal stress hormones in forming lasting memories in the brain. Curr Opin Neurobiol 12: 205-210.

Minson CT, Halliwill JR, Young TM, Joyner MJ. 2000. Influence of the menstrual cycle on sympathetic activity, baroreflex sensitivity, and vascular transduction in young women. Circulation 101: 862-868.

Nestler EJ, Barrot M, DiLeone RJ, Eisch AJ, Gold SJ, Monteggia LM. 2002. Neurobiology of depression. Neuron 34: 13-25.

Phillips SM, Sherwin BB. 1992. Variations in memory function and sex steroid hormones across the menstrual cycle. Psychoneuroendocrinology 17: 497-506.

Postma A, Winkel J, Tuiten A, van Honk J. 1999. Sex differences and menstrual cycle effects in human spatial memory. Psychoneuroendocrinology 24: 175-192.

Ressler KJ, Mayberg HS. 2007. Targeting abnormal neural circuits in mood and anxiety disorders: From the laboratory to the clinic. Nat Neurosci 10: $1116-1124$.

Reynolds M, Brewin CR. 1998. Intrusive cognitions, coping strategies and emotional responses in depression, posttraumatic stress disorder and a non-clinical population. Behav Res Ther 37: 201-215.

Roozendaal B. 2000. Glucocorticoids and the regulation of memory consolidation. Psychoneuroendocrinology 25: 213-238.

Rosenberg L, Park S. 2002. Verbal and spatial functions across the menstrual cycle in healthy young women. Psychoneuroendocrinology 27: $835-841$.

Segal SK, Cahill L. 2009. Endogenous noradrenergic activation and memory for emotional material in men and women. Psychoneuroendocrinology 34: 1263-1271.

Sherwin BB. 1994. Sex hormones and psychological functioning in postmenopausal women. Exp Gerontol 29: 423-430.
Shors T, Lewczyk C, Pacynski M, Mathew P, Pickett J. 1998. Stages of estrous mediate the stress-induced impairment of associative learning in the female rat. Neuroreport 9: 419-423.

Slabbekorn D, van Goozen SHM, Megens J, Gooren LJG,

Cohen-Kettenis PT. 1999. Activating effects of cross-sex hormones on cognitive functioning: A study of short-term and long-term hormone effects in transsexuals. Psychoneuroendocrinology 24: 423-447.

Stark R, Wolf OT, Tabbert K, Kagerer S, Zimmermann M, Kirsch P, Shienle A, Vaiti D. 2006. Influence of the stress hormone cortisol on fear condition in humans: Evidence for sex differences in the response of the prefrontal cortex. NeuroImage 32: 1290-1298.

Van Goozen SHM, Cohen-Kettenis PT, Gooren LJG, Frijda NH, Van De Poll NE. 1995. Gender differences in behaviour: Activating effects of cross-sex hormones. Psychoneuroendocrinology 20: 343-363.

van Stegeren AH. 2008. The role of the noraderenergic system in emotional memory. Acta Psychol (Amst) 127: 532-541.

van Stegeren AH, Wolf OT, Kindt M. 2008. Salivary $\alpha$ amylase and cortisol responses to different stress tasks: Impact of sex. Int J Psychophysiol 69: $33-40$.

van Wingen GA, van Broekhoven F, Verkes RJ, Petersson KM, Bäckström T, Buitelaar J, Fernández G. 2008a. How progesterone impairs memory for biologically salient stimuli in healthy young women. J Neurosci 27: 11416-11423.

van Wingen GA, van Broekhoven F, Verkes RJ, Petersson KM, Bäckström T, Buitelaar JK, Fernández G. 2008b. Progesterone selectively increases amygdala reactivity in women. Mol Psychiatry 13: 325-333.

Warren SG, Juraska JM. 1997. Spatial and nonspatial learning across the rat estrous cycle. Behav Neurosci 111: 259-266.

Yehuda R, Southwick SM, Nussbaum G, Wahby V, Giller EL Jr, Mason JW. 1990. Low urinary cortisol excretion in patients with posttraumatic stress disorder. J Nerv Ment Dis 178: 366-369.

Yehuda R, Boisoneau D, Lowy MT, Giller EL Jr. 1995. Dose-response changes in plasma cortisol and lymphocyte glucocorticoid receptors following dexamethasone administration in combat veterans with and without posttraumatic stress disorder. Arch Gen Psychiatry 52: 583-593.

Yehuda R, Teicher MH, Trestman RL, Levengood RA, Siever LJ. 1996. Cortisol regulation in posttraumatic stress disorder and major depression. Biol Psychiatry 40: 79-88.

Zorawski M, Blanding NQ, Kuhn CM, LaBar KS. 2006. Effects of stress and sex on acquisition and consolidation of human fear conditioning. Learn Mem 13: 441-450.

Received June 27, 2011; accepted in revised form October 6, 2011. 


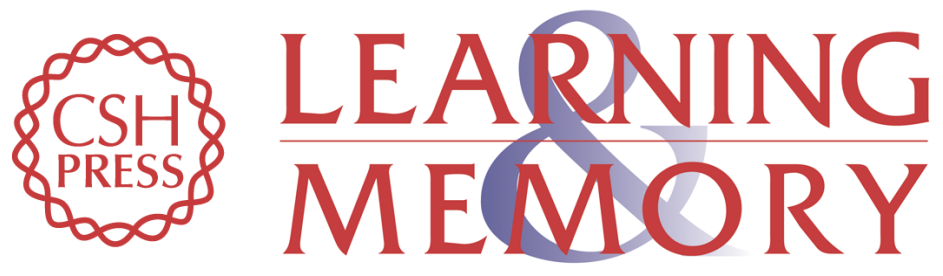

\section{Progesterone at encoding predicts subsequent emotional memory}

Nicole Ertman, Joseph M. Andreano and Larry Cahill

Learn. Mem. 2011, 18:

Access the most recent version at doi:10.1101//m.023267.111

References This article cites 57 articles, 8 of which can be accessed free at: http://learnmem.cshlp.org/content/18/12/759.full.html\#ref-list-1

License

Email Alerting Receive free email alerts when new articles cite this article - sign up in the box at the Service top right corner of the article or click here. 